PROCEEDINGS OF THE

AMERICAN MATHEMATICAL SOCIETY

Volume 134, Number 5, Pages 1365-1367

S 0002-9939(05)08104-9

Article electronically published on October 6, 2005

\title{
REMARKS ON A PAPER BY CHAO-PING CHEN AND FENG QI
}

\author{
STAMATIS KOUMANDOS
}

(Communicated by Carmen C. Chicone)

\begin{abstract}
In a recent paper, Chao-Ping Chen and Feng Qi (2005) established sharp upper and lower bounds for the sequence $P_{n}:=\frac{1.3 \ldots(2 n-1)}{2.4 \ldots 2 n}$. We show that their result follows easily from a theorem of G. N Watson published in 1959. We also show that the main result of Chen and Qi's paper is a special case of a more general inequality which admits a very short proof.
\end{abstract}

\section{INTRODUCTION AND RESULTS}

Let

$$
P_{n}:=\frac{1.3 \ldots(2 n-1)}{2.4 \ldots 2 n} .
$$

This sequence appears in Wallis's well-known product formula of approximation of $\pi$ and in various other topics of analysis and number theory. In the recent paper [2], Chao-Ping Chen and Feng Qi proved the following inequality:

$$
\frac{1}{\sqrt{\pi\left(n+\frac{4}{\pi}-1\right)}} \leq P_{n}<\frac{1}{\sqrt{\pi\left(n+\frac{1}{4}\right)}}, n=1,2, \ldots
$$

The constants $\frac{4}{\pi}-1$ and $\frac{1}{4}$ are the best possible. Inequality (1.1) improves some earlier results dealing with estimates of the sequence $P_{n}$.

Here we observe that (1.1) follows easily from a known result due to G. N. Watson [15. Indeed, it is shown in [15] that the function

$$
\theta(x)=\left(\frac{\Gamma(x+1)}{\Gamma\left(x+\frac{1}{2}\right)}\right)^{2}-x
$$

is strictly decreasing on $(-1 / 2, \infty)$. Applying this result, together with the observation that $\theta(1)=4 / \pi-1, \lim _{x \rightarrow \infty} \theta(x)=1 / 4$ and $P_{n}=\frac{\Gamma\left(n+\frac{1}{2}\right)}{\sqrt{\pi} \Gamma(n+1)}$, we obtain the double inequality (1.1).

The purpose of this note is to show that (1.1) is a special case of a more general inequality, which in fact, admits a very short proof. Let $0<\alpha<1$ and

$$
d_{n}(\alpha):=\frac{(1-\alpha)_{n}}{n !} .
$$

Received by the editors September 15, 2004 and, in revised form, November 30, 2004.

2000 Mathematics Subject Classification. Primary 33B15; Secondary 26D20.

Key words and phrases. Wallis' inequality, Gamma function, monotonicity, best bounds. 
As usual, $(a)_{k}$ denotes the Pochhammer symbol, defined by

$$
(a)_{0}=1, \text { and }(a)_{k}=a(a+1) \ldots(a+k-1)=\frac{\Gamma(k+a)}{\Gamma(a)}, \text { for } k=1,2, \ldots
$$

Clearly, $d_{n}(1 / 2)=P_{n}$. It is also well known and easy to see that

$$
\frac{1}{\Gamma(1-\alpha)(n+1)^{\alpha}}<d_{n}(\alpha)<\frac{1}{\Gamma(1-\alpha) n^{\alpha}} .
$$

Here we show that this inequality can be improved as follows.

Theorem 1.1. For all natural numbers $n$, we have

$$
\frac{1}{\Gamma(1-\alpha)\left(n+c_{2}\right)^{\alpha}} \leq d_{n}(\alpha)<\frac{1}{\Gamma(1-\alpha)\left(n+c_{1}\right)^{\alpha}},
$$

where the constants

$$
c_{1}=c_{1}(\alpha)=\frac{1-\alpha}{2} \quad \text { and } \quad c_{2}=c_{2}(\alpha)=\frac{1}{[\Gamma(2-\alpha)]^{1 / \alpha}}-1
$$

are the best possible.

It is clear that for $\alpha=1 / 2$, inequality (1.3) coincides with (1.1).

Inequalities such as (1.2) and (1.3) are of particular importance in certain problems on positive trigonometric sums and positive sums of Gegenbauer polynomials, having $d_{k}(\alpha)$ as a sequence of coefficients. See the recent papers [9] and [10]. We also refer to the paper [11] for some interesting estimates of ratios of gamma functions. Some other proofs of (1.1) and related results can be found in the recent articles [3], 4], 5], 6] and [7]. Compare also the papers [12], 13] and [14] for different proofs of (1.1) and various inequalities for the sequence $P_{n}$.

\section{Proof of (1.3)}

For the proof of (1.3) we define

$$
Q_{n}(\alpha):=\left\{\frac{\Gamma(n+1)}{\Gamma(n+1-\alpha)}\right\}^{1 / \alpha}-n
$$

Using the asymptotic formula

$$
x^{b-a} \frac{\Gamma(x+a)}{\Gamma(x+b)}=1+\frac{(a-b)(a+b-1)}{2 x}+O\left(\frac{1}{x^{2}}\right), x \rightarrow \infty
$$

(see [1, p. 615, for the complete form of this formula), we easily verify that

$$
\lim _{n \rightarrow \infty} Q_{n}(\alpha)=\frac{1-\alpha}{2}=c_{1} .
$$

Obviously $c_{2}=Q(1, \alpha)$.

The required inequality (1.3) follows from the fact that the sequence $Q_{n}(\alpha)$ is strictly decreasing. This, in turn, is an immediate consequence of a result of N. Elezović, C. Giordano and J. Pečarić, 8] who showed that the function

$$
x \mapsto\left(\frac{\Gamma(x+t)}{\Gamma(x+s)}\right)^{1 /(t-s)}-x
$$

is convex and decreasing on $(-r, \infty)$ if $|t-s|<1$, where $s, t$ are given positive numbers and $r=\min (s, t)$.

This completes the proof of (1.3). 


\section{REFERENCES}

[1] G. E. Andrews, R. Askey, and R. Roy, Special functions, Encyclopedia of Mathematics and its Applications, 71. Cambridge University Press, Cambridge, 1999. xvi+664 pp. ISBN: 0-521-62321-9; 0-521-78988-5 Cambridge University Press, Cambridge, 1999. MR1688958 (2000g:33001)

[2] Chao-Ping Chen and Feng Qi, The best bounds in Wallis' inequality, Proc. Amer. Math. Soc. 133 (2005), no. 2, 397-401. MR2093060

[3] Chao-Ping Chen and Feng Qi, Best upper and lower bounds in Wallis' inequality, J. Indones. Math. Soc. 12 (2006), to appear.

[4] Chao-Ping Chen and Feng Qi, Completely monotonic function associated with the gamma function and proof of Wallis' inequality, Tamkang J. Math. 36, (2005), no. 4, to appear.

[5] Chao-Ping Chen and Feng Qi, The best bounds to $\frac{(2 n) !}{2^{2 n}(n !)^{2}}$, Math. Gaz. 88 (2004), 54-55.

[6] Chao-Ping Chen and Feng Qi, Improvement of lower bound in Wallis' inequality, RGMIA Res. Rep. Coll. 5 (2002), suppl., Art. 23. Available online at http://rgmia.vu.edu.au/v5(E).html.

[7] Chao-Ping Chen and Feng Qi, A new proof of the best bounds in Wallis' inequality, RGMIA Res. Rep. Coll. 6 (2003), no. 2, Art. 2. Available online at http://rgmia.vu.edu.au/v6n2.html.

[8] N. Elezović, C. Giordano and J. Pečarić, The best bounds in Gautschi's inequality, Math. Inequal. Appl. 3 (2000), no. 2, 239-252. MR1749300 (2001g:33001)

[9] S. Koumandos and S. Ruscheweyh, Positive Gegenbauer polynomial sums and applications to starlike functions, Constr. Approx., to appear.

[10] S. Koumandos, An extension of Vietoris's inequalities, Ramanujan J., to appear.

[11] S. -L. Qiu and M. Vuorinen, Some properties of the gamma and psi functions, with applications, Math. Comp. 74 (2005), 723-742.

[12] Z. Sasvári, Inequalities for binomial coefficients, J. Math. Anal. Appl. 236 (1999), no. 1, 223-226. MR 1702663(2000d:26024)

[13] D. V. Slavić, On inequalities for $\Gamma(x+1) / \Gamma(x+1 / 2)$, Univ. Beograd. Publ. Elektrotehn. Fak. Ser. Mat. Fiz. no. 498-541 (1975), 17-20. MR0385182 (52:6047)

[14] P. Stănică, Good lower and upper bounds on binomial coefficients, JIPAM. J. Inequal. Pure Appl. Math. 2 (2001), no. 3, Article 30, 5 pp. (electronic). MR.1876263 (2003g:05018)

[15] G. N. Watson, A note on gamma functions, Proc. Edinburgh Math. Soc. (2) 11 (1958/1959), Edinburgh Math. Notes 42 (1959), 7-9. MR0117358 (22:8138)

Department of Mathematics and Statistics, University of Cyprus, P.O. Box 20537, 1678 Nicosia, Cyprus

E-mail address: skoumand@ucy.ac.cy 